Pacific Journal of Mathematic 


\title{
A COMBINATORIAL PROBLEM IN THE SYMMETRIC GROUP
}

\author{
OSCAR Rothaus and John G. Thompson
}

If $G$ is a group and $T$ is a nonempty subset of $G$, we say that $T$ divides $G$ if and only if $G$ contains a subset $S$ such that every element of $G$ has a unique representation as $t s$ with $t$ in $T, s$ in $S$, in which case we write $T \cdot S=G$. We study the case where $G$ is $\Sigma_{n}$, the symmetric group on $n$ symbols and $T$ is the set consisting of the identity and all transpositions in $\Sigma_{n}$.

The problem may be given a combinatorial setting as follows: For $x, y$ in $\Sigma_{n}$, let $d(x, y)$ be the minimum number of transpositions needed to write $x y^{-1}$. One verifies that $d$ converts $\Sigma_{n}$ into a metric space, and that $T$ divides $\Sigma_{n}$ if and only if $\Sigma_{n}$ can be covered by disjoint closed spheres of radius one.

We use the irreducible characters of $\Sigma_{n}$, together with judiciously selected permutation representations of $\Sigma_{n}$, to prove the following result.

Theorem. If $1+(n(n-1)) / 2$ is divisible by a prime exceeding $\sqrt{n}+2$, then $T$ does not divide $\Sigma_{n}$.

The proof depends on properties of $\Sigma_{n}$ (see [1] and [2], pp. 190-193).

If $\lambda_{1}, \lambda_{2}, \cdots, \lambda_{s}$ are the parts of the partition $\sigma$ in decreasing order and $\mu_{1}, \cdots, \mu_{t}$ are the parts of the partition $\tau$ in decreasing order, we write $\sigma>\tau$ provided the first nonvanishing difference $\lambda_{i}-\mu_{i}$ is positive. We say that $\sigma$ dominates $\tau$ provided $\lambda_{i}-\mu_{i} \geqq 0$ for $i=$ $1,2, \cdots, s$. Let $\sigma^{\prime}$ be the conjugate partition to $\sigma$ with parts $\lambda_{1}^{\prime} \geqq$ $\lambda_{2}^{\prime} \geqq \cdots \geqq \lambda_{s^{\prime}}^{\prime}$, and set

$$
\pi(\sigma)=\sum_{i=1}^{s} \frac{\lambda_{i}\left(\lambda_{i}-1\right)}{2}-\sum_{i=1}^{s^{\prime}} \frac{\lambda_{i}^{\prime}\left(\lambda_{i}^{\prime}-1\right)}{2} .
$$

The function $\pi$ has a simple interpretation. Namely, in the dot diagram of $\sigma$, the number of unordered pairs of dots in a common row minus the number of unordered pairs of dots in a common column equals $\pi(\sigma)$. However, it will become apparent that $\pi(\sigma)$ has a group theoretic interpretation too.

Lemma 1. If $\sigma$ dominates $\tau$, and $\sigma \neq \tau$ then $\pi(\sigma)>\pi(\tau)$.

Proof. Let the parts of $\sigma$ be $\lambda_{1} \geqq \lambda_{2} \geqq \cdots \geqq \lambda_{s}$ and those of $\tau$ be $\mu_{1} \geqq \mu_{2} \geqq \cdots \geqq \mu_{t}$. By the hypotheses, we may suppose that $\lambda_{1}=\mu_{1}$, $\lambda_{2}=\mu_{2}, \cdots, \lambda_{r-1}=\mu_{r-1}, \lambda_{r}>\mu_{r}$ and $\lambda_{r+1} \geqq \mu_{r+1}, \cdots, \lambda_{s} \geqq \mu_{s}$, for some 
integer $r$ less than $s$. A straightforward computation shows that $\pi(\tau)$ increases if $\mu_{r}$ is increased by 1 and $\mu_{t}$ is decreased by 1 . With this observation made, the result is clear.

For any subset $A$ of $\Sigma_{n}, \dot{A}$ denotes the sum of the elements of $A$ in the group algebra of $\Sigma_{n}$ (over the rationals), while

$$
\ddot{A}=\sum_{a \in A} s g(a) \cdot a \text {. }
$$

LEMma 2. If $R=R_{\sigma}$ is the irreducible representation of $\Sigma_{n}$ associated to the partition $\sigma$, then $R(\dot{T})$ is singular if and only if $\pi(\sigma)=1$.

Proof. Since $\dot{T}$ is in the center of the group algebra, $R(\dot{T})$ is a scalar matrix, say $(1+c) I=R(\dot{T})$. Thus, $R(\dot{T})$ is singular if and only if $c=-1$. Let $Y$ be a Young tableau associated to $\sigma$, that is, the dot diagram of $\sigma$ with a label on each dot, the labels coming from and exhausting the set $\{1,2, \cdots, n\}$. Let $A$ be the subgroup of $\Sigma_{n}$ permuting the columns of $Y$ and $B$ the subgroup of $\Sigma_{n}$ permuting the rows of $Y$, and let

$$
E=\frac{\dot{A}}{|A|} \cdot \frac{\ddot{B}}{|B|} .
$$

Then $E$ is a primitive idempotent and has the property that $R_{\tau}(E)=0$ for $\tau \neq \sigma$. We have $R_{\sigma}(E) R_{\sigma}(\dot{T})=(1+c) R_{\sigma}(E)$. As $E$ vanishes in each $R_{\tau}$ with $\tau \neq \sigma$, we have trivially, $R_{\tau}(E) \cdot R_{\tau}(\dot{T})=(1+c) R_{\tau}(E)$ for all $\tau \neq \sigma$. Hence

$$
E \cdot \dot{T}=(1+c) E .
$$

Let $T_{0}$ be the set of transpositions in $\Sigma_{n}$. Then (4) implies

$$
E \cdot \dot{T}_{0}=c E \text {. }
$$

Since $A \cap B=1$, to determine $c$, it suffices to determine the multiplicity (i.e., coefficient) of 1 in $E \cdot \dot{T}_{0}$. It follows readily that $c=$ $\Sigma s g(b)$, the summation ranging over all triples $(a, b, t)$ with $a$ in $A, b$ in $B, t$ in $T_{0}$, such that $a b t=1$. Since $a b t=1$ if and only if $a b=t$, it is easy to see that whenever $a b t=1$, then either $t \in A$ or $t \in B$. Hence, $c=-\pi(\sigma)$, as required.

In the following discussion, $\sigma, Y, A, B, E$ have the same meaning as above.

We next consider a family of permutation representations of $\Sigma_{n}$. Let $X$ be a Young tableau for the partition $\tau$ and let $C$ be the subgroup permuting the columns of $X$. Then $P_{\tau}$ denotes the permutation representation of $\Sigma_{n}$ on the cosets of $C$. Thus, for $x$ in $\Sigma_{n}$, 
$P_{\tau}(x): C g \rightarrow C g x$. It is clear that $P_{\tau}$ depends only on $\tau$ and not on $X$. As is customary, we view $P_{\tau}$ as a representation of the group algebra.

Lemma 3. If $\sigma>\tau$, then $R_{\sigma}$ is not a constituent of $P_{\tau}$.

Proof. Since $E$ is a primitive idempotent, $\operatorname{tr}\left(P_{\tau}(E)\right)$ is the multiplicity of $R_{\sigma}$ in $P_{\tau}$. Consider a coset $C g$. A contribution to $\operatorname{tr}\left(P_{\tau}(E)\right)$ occurs each time $C g a b=C g$ with $a$ in $A, b$ in $B$, the contribution being

$$
\frac{s g(b)}{|A| \cdot|B|}
$$

Thus, from the coset $C g$, we get

$$
\frac{\Sigma s g(b)}{|A| \cdot|B|}
$$

the summation being over those pairs $(a, b)$ with $a$ in $A, b$ in $B$ and $a b$ in $g^{-1} C g$. As $\sigma>\tau$, it is easy to verify that there is a row of $Y$ which has at least two symbols in common with some column of $X g$, that is, $B \cap g^{-1} C g$ contains a transposition $t=t(g)$. This implies that whenever a pair $(a, b)$ occurs in the above summation, so does the pair $(a, b t)$, so $\operatorname{tr}\left(P_{\tau}(E)\right)=0$, as required.

Now let $p$ be a prime divisor of $1+(n(n-1)) / 2$ with $p \geqq \sqrt{n}+2$. Let $n=(p-1) q+r$ with $0 \leqq r<p-1$. Hence $q<p-2$. Let $\tau$ be the partition of $n$ with $r$ parts equal to $q+1$ and $p-1-r$ parts equal to $q$. We see that $\tau^{\prime}$ has $q$ parts equal to $p-1$ and one part equal to $r$. Hence

$$
\begin{aligned}
\pi(\tau)= & \frac{(q+1) q}{2} r+\frac{q(q-1)}{2}(p-1-r) \\
& -\left\{\frac{(p-1)(p-2)}{2} q+\frac{r(r-1)}{2}\right\} \\
= & \frac{q(p-1)}{2}\{q+1-(p-2)\}-\frac{r(r-1)}{2}-q(p-1-r) .
\end{aligned}
$$

Since $q+1 \leqq p-2$, it follows that $\pi(\tau)<-1$.

By Lemma 3 , if $R_{\sigma}$ is a constituent of $P_{\tau}$, then $\sigma \leqq \tau$. The structure of $\tau$ now yields that whenever $\sigma \leqq \tau$, then $\tau$ dominates $\sigma$.

By Lemma $1, \pi(\sigma) \leqq \pi(\tau)<-1$, and hence by Lemma $2, R_{\sigma}(\dot{T})$ is nonsingular. Thus $P_{\tau}(\dot{T})$ is nonsingular.

Let $d=d_{\tau}$ be the degree of $P_{\tau}$. Since $d=\left|\Sigma_{n}: C\right|$, we see that $d$ is divisible by the same power of $p$ as $\left|\Sigma_{n}\right|$, since $|C|=(p-1) !^{q} r$ ! is prime to $p$. Now suppose $T \cdot U=\Sigma_{n}$. Then $P_{\tau}(\dot{T}) P_{\tau}(U)=P_{\tau}\left(\dot{\Sigma}_{n}\right)$. 
It is clear that $P_{\tau}\left(\dot{\Sigma}_{n}\right)$ is the matrix with $|C|$ in every entry, so is of rank 1. Since $P_{\tau}(\dot{T})^{-1}$ is a polynomial in $P_{\tau}(\dot{T})$, and since $P_{\tau}\left(\dot{\Sigma}_{n}\right)=$ $P_{\tau}(\dot{x}) P_{\tau}\left(\dot{\Sigma}_{n}\right)$ for all $x$ in $\Sigma_{n}$, it follows that $P_{\tau}(\dot{U})=a P_{\tau}\left(\dot{\Sigma}_{n}\right)$ for some rational number $a$. This implies that $a(1+(n(n-1)) / 2)=1$, so that

$$
P_{\tau}(\dot{U})=\frac{1}{1+\frac{n(n-1)}{2}} P_{\tau}\left(\dot{\Sigma}_{n}\right)
$$

does not have integral entries, which is a contradiction, since $P_{\tau}(\dot{U})$ is a sum of $|U|$ permutation matrices.

REMARK 1. The integers $1,2,3,6,91,137,733$ and 907 are the only integers less than 1,000 which fail to satisfy the theorem.

REMARK 2. As the referee has noted, essentially the same proof yields: If $(n(n-1)) / 2$ is divisible by a prime exceeding $\sqrt{n}+2$, then $T_{0}$ does not divide $\Sigma_{n}$.

\section{REFERENCES}

1. D. E. Littlewood, The theory of group characters, Oxford at the Clarendon Press.

2. B. L. van der Waerden, Modern algebra, Vol. II.

Received December 22, 1964.

InSTITUte FOR DEFEnSE ANALYSES

Princeton, New Jersey

UNIVERSITY OF CHICAGO 


\section{PACIFIC JOURNAL OF MATHEMATICS}

H. SAMELSON

Stanford University Stanford, California

R. M. BLUMENTHAL

University of Washington

Seattle, Washington 98105

\section{EDITORS}

\author{
*J. DugundJI \\ University of Southern California \\ Los Angeles, California 90007 \\ RICHARD ARENS \\ University of California \\ Los Angeles, California 90024
}

\section{ASSOCIATE EDITORS}

E. F. BeCKENBACH

B. H. NEUMANN

F. WOLF

K. YosidA

\section{SUPPORTING INSTITUTIONS}

UNIVERSITY OF BRITISH COLUMBIA

CALIFORNIA INSTITUTE OF TECHNOLOGY

UNIVERSITY OF CALIFORNIA

MONTANA STATE UNIVERSITY

UNIVERSITY OF NEVADA

NEW MEXICO STATE UNIVERSITY

OREGON STATE UNIVERSITY

UNIVERSITY OF OREGON

OSAKA UNIVERSITY

UNIVERSITY OF SOUTHERN CALIFORNIA
STANFORD UNIVERSITY

UNIVERSITY OF TOKYO

UNIVERSITY OF UTAH

WASHINGTON STATE UNIVERSITY

UNIVERSITY OF WASHINGTON

AMERICAN MATHEMATICAL SOCIETY CHEVRON RESEARCH CORPORATION TRW SYSTEMS

NAVAL ORDNANCE TEST STATION 


\section{Pacific Journal of Mathematics}

Vol. 18, No. 1

March, 1966

Edward Joseph Barbeau, Semi-algebras that are lower semi-lattices ...... 1

Steven Fredrick Bauman, The Klein group as an automorphism group

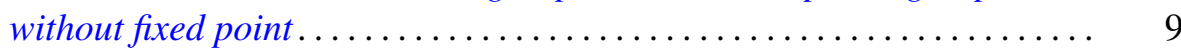

Homer Franklin Bechtell, Jr., Frattini subgroups and $\Phi$-central groups .... 15

Edward Kenneth Blum, A convergent gradient procedure in prehilbert

spaces ............................................

Edward Martin Bolger, The sum of two independent exponential-type random variables ...................................

David Wilson Bressler and A. P. Morse, Images of measurable sets .......

Dennison Robert Brown and J. G. LaTorre, A characterization of uniquely

divisible commutative semigroups........................

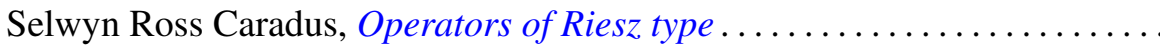

Jeffrey Davis and Isidore Isaac Hirschman, Jr., Toeplitz forms and ultraspherical polynomials ............................

Lorraine L. Foster, On the characteristic roots of the product of certain rational integral matrices of order two ......................

Alfred Gray and S. M. Shah, Asymptotic values of a holomorphic function

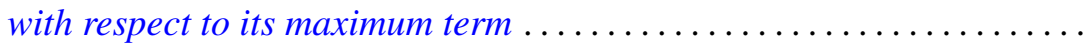

Sidney (Denny) L. Gulick, Commutativity and ideals in the biduals of

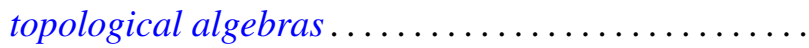

G. J. Kurowski, Further results in the theory of monodiffric functions

Lawrence S. Levy, Commutative rings whose homomorphic images are self-injective .

Calvin T. Long, On real numbers having normality of order $k$....

Bertram Mond, An inequality for operators in a Hilbert space. ...

John William Neuberger, The lack of self-adjointness in three-point boundary value problems ........................

C. A. Persinger, Subsets of $n$-books in $E^{3}$

Oscar S. Rothaus and John Griggs Thompson, A combinatorial problem in the symmetric group ............................... 175

Rodolfo DeSapio, Unknotting spheres via Smale .................. 179

James E. Shockley, On the functional equation

$$
F(m n) F((m, n))=F(m) F(n) f((m, n)) \ldots \ldots \ldots
$$

Kenneth Edward Whipple, Cauchy sequences in Moore spaces ... 